University of Nebraska - Lincoln

DigitalCommons@University of Nebraska - Lincoln

September 1970

\title{
de Haas-van Alphen Effect in Dilute Beryllium-Copper Alloys
}

I.S. Goldstein

Center for Materials Science and Engineering and Department of Metallurgy and Materials Science, Massachusetts Institute of Technology, Cambridge, Massachusetts

David J. Sellmyer

University of Nebraska-Lincoln, dsellmyer@unl.edu

B.L. Averbach

Center for Materials Science and Engineering and Department of Metallurgy and Materials Science, Massachusetts Institute of Technology, Cambridge, Massachusetts

Follow this and additional works at: https://digitalcommons.unl.edu/physicssellmyer

Part of the Physics Commons

Goldstein, I.S.; Sellmyer, David J.; and Averbach, B.L., "de Haas-van Alphen Effect in Dilute BerylliumCopper Alloys " (1970). David Sellmyer Publications. 181.

https://digitalcommons.unl.edu/physicssellmyer/181

This Article is brought to you for free and open access by the Research Papers in Physics and Astronomy at DigitalCommons@University of Nebraska - Lincoln. It has been accepted for inclusion in David Sellmyer Publications by an authorized administrator of DigitalCommons@University of Nebraska - Lincoln. 
Eq. (4), is obtained.

We conclude by noting that the origin of a major contribution to the $g$ shift of conduction electrons in sodium metal (in our calculation) lies in the difference between the orbital parts $R_{1 / 2}$ and $R_{3 / 2}$ of the $2 p$ core wave functions, caused by the spin-orbit splitting of the $j=\frac{1}{2}$ and $j=\frac{3}{2} 2 p$ core states. This leads to a conduction-electron $g$ shift proportional to the difference in amplitude of these core functions in the orthogonalized plane wave. This is most clearly expressed by Eqs. (23) and (24).

\footnotetext{
${ }^{1}$ Y. Yafet, Phys. Rev. 106, 679 (1957); Solid State Phys. 14, 1 (1963).

${ }^{2}$ L. M. Roth, J. Phys. Chem. Solids 23, 433 (1962)。

${ }^{3}$ E. I. Blount, Phys. Rev. 126, 1636 (1962).

${ }^{4}$ A. M. de Graaf and A. W. Overhause, Phys. Rev. 180, 701 (1969); A. W. Overhauser and A. M. de Graaf, Phys. Rev. Letters 22, 127 (1969).

${ }^{5}$ Y. Yafet, Phys。 Rev. 85, 478 (1952).

${ }^{6}$ H. Brooks, Phys. Rev. 94, A1411 (1954).

${ }^{7} \mathrm{~A}$. Bienenstock and H. Brooks, Phys。Rev. 136, A784 (1964).

${ }^{8}$ Actually, Bienenstock and Brook's expression for $(\delta g)^{\prime}$ is too large by a factor of 2 . The value $0.6 \times 10^{-5}$ is half their quoted value.

${ }^{9}$ G. J. King, B. S. Miller, F. F. Carlson, and R. C.
}

Mc Millan, J. Chem. Phys. 32, 940 (1960).

${ }^{10}$ G. Feher and A. F. Kip, Phys。 Rev. 98, 337 (1955);

Ch. Ryter, Phys. Letters 4,69 (1963)。

${ }^{11}$ I. H. Solt, Jr., and M. W. P. Strandberg, Phys. Rev. 95, A607 (1954).

${ }^{12}$ E. Merzbacher, Quantum Mechanics (Wiley, New York, 1961), p. 402.

${ }^{13} \mathrm{~J}$. A. Bearden and A. F. Burr, Rev. Mod. Phys. 39, 125 (1967).

${ }^{14}$ J. Dickey, Phys. Rev. 81, 612 (1951).

${ }^{15} \mathrm{C}$. E. Moore, Atomic Energy Levels (Natl. Bur. Stds., Washington, D. C., 1949), Circular No. 467, Vol. 1.

${ }^{16}$ C. S. Barrett, Acta Cryst. 9, 671 (1956).

\title{
de Haas-van Alphen Effect in Dilute Beryllium-Copper Alloys*
}

\author{
I. S. Goldstein, † D. J. Sellmyer, and B. L. Averbach \\ Center for Materials Science and Engineering and Department of Metallurgy and Materials Science, \\ Massachusetts Institute of Technology, Cambridge, Massachusetts 02139
}

(Received 19 February 1970)

\begin{abstract}
The de Haas -van Alphen effect was investigated in pure beryllium and in $\mathrm{Be}-\mathrm{Cu}$ alloys containing up to 0.34 at. $\% \mathrm{Cu}$. The extremal cross-sectional areas of the cigar-shaped thirdband electron pieces of Fermi surface decreased with alloying. The areas of the coronetshaped second-band hole piece increased with alloying. The magnitude of the area changes were compared with rigid-band-theory predictions. Corrections for lattice-parameter changes were included when possible. In the low-concentration limit, the theory is able to account for the observed area changes. For the highest-concentration alloy, there are deviations, for all areas measured, to greater changes than are predicted by the theory. However, further data in the higher-concentration region $(\gtrsim 0.3$ at. \% copper) are needed to verify this trend. Amplitude measurements were made to obtain cyclotron masses and scattering temperatures. Within experimental error, the masses were found to be independent of alloying. A significant anisotropy over the Fermi surface was observed in the rate of change of scattering temperature with respect to copper concentration. An analysis was made to determine the scattering rate due to copper impurities for the four orbits studied. The results indicate that this scattering rate is considerably larger for the two cigar orbits than it is for the two coronet orbits. The relative values of the scattering times as determined by the de Haas-van Alphen effect and residual-resistivity measurements were compared with the free-electron theory of Brailsford. Only order-of-magnitude agreement was found.
\end{abstract}

\section{INTRODUCTION}

The rigid-band model (RBM) as introduced by Jones ${ }^{1}$ was the first attempt to explain the change in the electronic state of a pure metal upon alloying. This model proposed that the effect of alloying was to change the electron density by just the difference between the valence of the solute atom and the solvent atom, scaled by the atom fraction of solute. Later Friedel $^{2}$ used simple perturbation theory to show that the screening of the solute ions produces a shift of all the energy states of the electrons. In a first approximation this shift is independent of the wave vector and the 
conduction band is displaced rigidly, thus, giving results similar to the RBM proposed by Jones. More recently, Stern, in a series of papers, discussed charging effects in the vicinity of solute atoms and gave criteria for the validity of the RBM. ${ }^{3-6}$

On the other hand, experimental studies of electron states in alloys have been limited in the main to classical experiments such as the Hall effect, thermoelectric power, electronic specific heat, and optical properties. The usual Fermi-surface techniques, which have played such an important role in advancing our understanding of pure metals, are inapplicable to concentrated alloys except for highly ordered compounds. However, it is possible to use the de Haas-van Alphen (dHvA) effect, for example, to investigate dilute alloys by employing very high magnetic fields.

There are two ways in which the addition of impurities alters the dHvA effect in a metal. First, the additional scattering of the conduction electrons diminishes the amplitude of the effect. Second, the energy levels shift and their populations change in such a way that the Fermi surface in the alloy has a different size and shape from that in the pure metal. Thus, the dHvA frequencies change on alloying. Theoretical treatments of the dHvA effect in alloys have attempted to treat both the scattering aspects as well as the changes in Fermi surface. Brailsford, ${ }^{7}$ building upon the earlier work of Dingle, ${ }^{8}$ showed how an impurity relaxation time $\tau$ appeared in the amplitude of the dHvA effect. He also showed how $\tau$ was related to the conductivity relaxation time $\tau_{\rho}$. The changes in dHvA frequency on alloying were first considered by Heine, ${ }^{9}$ who based his work on the RBM theory of Friedel. There have been no attempts to calculate the energy-dispersion curves in a specific solid-solution alloy, and thus obtain the shape of the Fermi surface.

Recently, there have been several dHvA investigations in dilute alloys. These have included alloys based on the noble metals, ${ }^{10,11}$ semimetals, ${ }^{12,13}$ and several polyvalent metals. ${ }^{14-18}$ While the RBM appears to hold qualitatively for the cubic metals with nontransition-metal solutes, the agreement for hexagonal metal alloys is rather poor. For example, Higgins and Marcus ${ }^{14}$ found that the change in lattice parameters on alloying had to be considered to explain the deviation of $\mathrm{Zn}$ from RBM behavior. However, this analysis depended on the proximity of the Fermi surface of $\mathrm{Zn}$ to the nearly-free electron (NFE) model.

Beryllium deviates much more from the NFE model than either zinc or magnesium. It is also interesting because it has a very low atomic number (4) and is one of the few hexagonal metals with a lower than ideal $c / a$ ratio (1.5677). ${ }^{19}$ The Fermi surface of beryllium has been calculated using the orthogonalized-plane-wave (OPW) method m,21 $^{20}$ and the augmented-plane-wave (APW) method. ${ }^{22}$ In addition, a nonlocal pseudopotential model has been constructed to fit experimental dHvA data. ${ }^{23}$ Watts $^{24}$ carried out the first extensive experimental measurements on the Fermi surface of $\mathrm{Be}$ and this has been followed by several other quantumoscillatory ${ }^{23,25,26}$ and magnetoresistance ${ }^{27-29}$ investigations.

The purpose of the present investigation was to investigate three aspects of the electronic structure and electron scattering when beryllium was alloyed with copper. (i) Measurements were made of the changes in several Fermi-surface cross sections to further test the RBM in hexagonal metals. (ii) An attempt was made to measure changes in cyclotron masses of various orbits on alloying. (iii) Anisotropic effects were investigated in the charged impurity-conduction electron scattering and the relaxation times for several orbits on the Fermi surface were compared with those predicted by the theory of Brailsford. ${ }^{7}$

In Sec. II, a brief discussion of the experimental techniques is given. The experimental results and discussion of each of the above three topics is given in a separate part of Sec. III, along with a brief description of some anomalous amplitude effects which were observed. Section IV consists of a summary and conclusions.

\section{EXPERIMENTAL PROCEDURE}

The dHvA experiments were performed at the Francis Bitter National Magnet Laboratory using 150-kG water-cooled solenoids. These solenoids were periodically recalibrated, using a coil and integrator, to an accuracy of $0.2 \%$. The coil and integrator were calibrated by the Magnet Laboratory staff using NMR techniques. In order to improve the field stability of the solenoids a superconducting shield was used around the sample holder. This device acted as a low-pass filter and has been described in detail elsewhere. ${ }^{30}$

The dHvA sample holder, which has been described previously, ${ }^{31}$ was similar to one constructed by Thorsen and Berlincourt. ${ }^{32}$ The field-modulation technique ${ }^{33}$ was used throughout with a modulation frequency of $100 \mathrm{~Hz}$ and lock-in detection at $200 \mathrm{~Hz}$. Modulation amplitudes of approximately 200-G peak-to-peak were used. dHvA field sweeps were taken with both increasing and decreasing fields and the dHvA peak positions were measured to within $\pm 160 \mathrm{G}$.

The single crystals used in this investigation were grown at the Franklin Institute. They were in the shape of rods with a square cross section 
approximately $0.13 \mathrm{~cm}$ on a side and 0.63 to 1.0 $\mathrm{cm}$ in length. The composition, accurate to approximately \pm 0.01 at. $\% \mathrm{Cu}$, and general orientation of the long axis of these samples are summarized in Table I. Further details of the growth and preparation of the samples are given elsewhere. ${ }^{34}$

\section{RESULTS AND DISCUSSION}

\section{A. Extremal Cross-Sectional Areas}

Figure 1(a) shows the Fermi surface of Be as well as the extremal cross-sectional areas which were measured. The $\alpha$ frequencies were measured with the field $\overrightarrow{\mathrm{B}} \|[0001]$, while, for the $\beta$ and $\gamma$ frequencies, $\vec{B} \|[11 \overline{2} 0]$. The orientation of the Fermi surface in the Brillouin zone is shown in Fig 1(b). The frequencies corresponding to these areas were obtained by measuring the field at dHvA peaks spaced at appropriate intervals. The peaks were chosen at intervals ranging from every other oscillation to every seventeenth oscillation depending on frequency. A linear leastsquares fit of reciprocal-field versus peak integer was used to obtain the frequency values. The fractional change in frequency versus copper concentration for the $\alpha_{2}, \beta$, and $\gamma$ oscillations is given in Fig. 2. The $\alpha_{1}$ and $\alpha_{2}$ frequencies produced beats with $\alpha_{2}$, the dominant frequency. Since, within experimental error, no change in the beat frequency was detected as a function of concentration, the concentration dependence of $\alpha_{1}$ is similar to that of the $\alpha_{2}$ oscillation. The error bars shown in Fig. 2 include both the random and systematic errors. The random error was defined using two standard deviations from the mean value. The systematic error was found by recalculation of the frequencies using estimated absolute errors in the field calibration of plus and minus $0.2 \%$.

The solid line in each of these graphs was calculated from the equation

$$
\Delta F / F=\left(Z / 2 \mu_{B} F\right)\left[m_{c} / n\left(E_{F}\right)\right] X
$$

TABLE I. Beryllium crystal samples.

\begin{tabular}{|c|c|c|c|}
\hline Notation & $\begin{array}{l}\text { Method of } \\
\text { preparation }\end{array}$ & $\begin{array}{l}\text { at. } \% \\
\mathrm{Cu}\end{array}$ & Orientation \\
\hline$B 0$ & $\begin{array}{l}\text { distilled and } \\
\text { zone refined }\end{array}$ & 0 & $c$ axis only \\
\hline$B 1$ & zone refined & 0 & $\begin{array}{l}c \text { axis and } \\
\text { basal plane }\end{array}$ \\
\hline$B 2$ & zone refined & 0.097 & $\begin{array}{l}c \text { axis and } \\
\text { basal plane }\end{array}$ \\
\hline$B 3$ & zone refined & 0.16 & $\begin{array}{l}c \text { axis and } \\
\text { basal plane }\end{array}$ \\
\hline$B 4$ & zone refined & 0.34 & $\begin{array}{l}c \text { axis and } \\
\text { basal plane }\end{array}$ \\
\hline
\end{tabular}

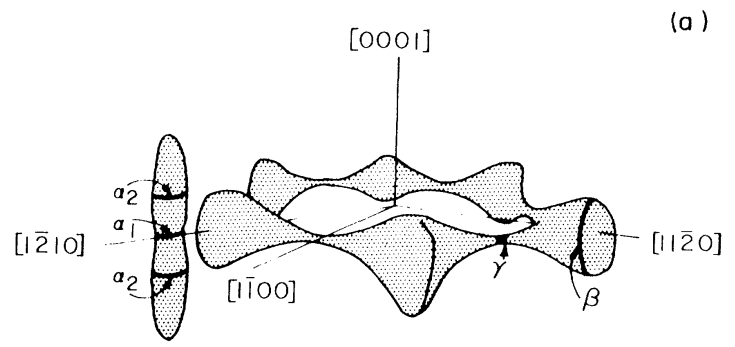

(b)

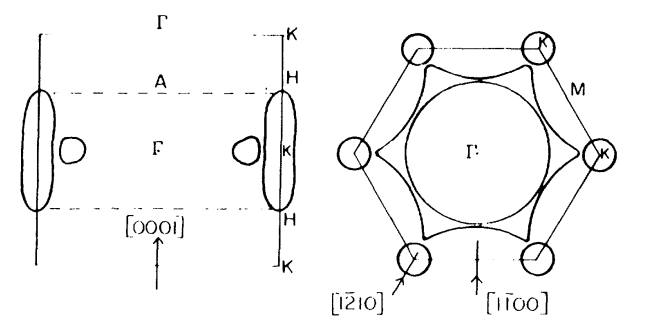

FIG. 1. (a) Fermi surface of Be showing $\alpha_{1}, \alpha_{2}, \beta$, and $\gamma$ orbits. (b) Two sections through the double Brillouin zone with the Fermi surface drawn approximately to scale (after Refs. 21 and 24).

derived from the RBM approximation as discussed by Heine. ${ }^{9}$ In this equation $F$ is the frequency for the pure material, $\Delta F$ is the change in frequency for an $X$ atomic-fraction copper concentration, $Z$ is the valence difference between the solute ( 1 for $\mathrm{Cu}$ ) and solvent (2 for $\mathrm{Be}$ ), $\mu_{B}$ is the Bohr magneton, $m_{c}$ is the cyclotron mass in units of the free-electron mass, and $n\left(E_{F}\right)$ is the density of states at the Fermi level. The cyclotron mass and density of states used in Eq. (1) were those obtained from the band-structure calculation of Tripp et $a l .{ }^{23}$ rather than experimentally determined values of $m_{c}$ and $n\left(E_{F}\right)$ obtained from dHvA effect and specific-heat measurements. This was done because the experimentally measured $m_{c}$ and $n\left(E_{F}\right)$ values are known to be enhanced by manybody interactions, especially electron-phonon interactions. ${ }^{35}$ However, since $m_{c}$ and $n\left(E_{F}\right)$ are enhanced equally, ${ }^{36}$ the ratio $m_{c} / n\left(E_{F}\right)$ should be the same whether calculated with experimentally determined values or band-structure values. Now, $\left[n\left(E_{F}\right)\right]_{\text {exp t }}=0.99$ states $/($ atom Ry $),{ }^{37}$ while $\left[n\left(E_{F}\right)\right]_{\mathrm{band}}=0.85$ states $/(\text { atom Ry })^{23}$; the ratio of these two quantities is 1.16 which does, in fact, agree roughly with the values for $\left[m_{c}\right]_{\text {expt }} /\left[m_{c}\right]_{\text {band }}$, which lie in the range from 1.18 to $1.24 .{ }^{23}$ In any case, it is clear from Fig. 2 that the experimental results for all three frequencies agree rather well with the RBM results shown by the lines in the low- 

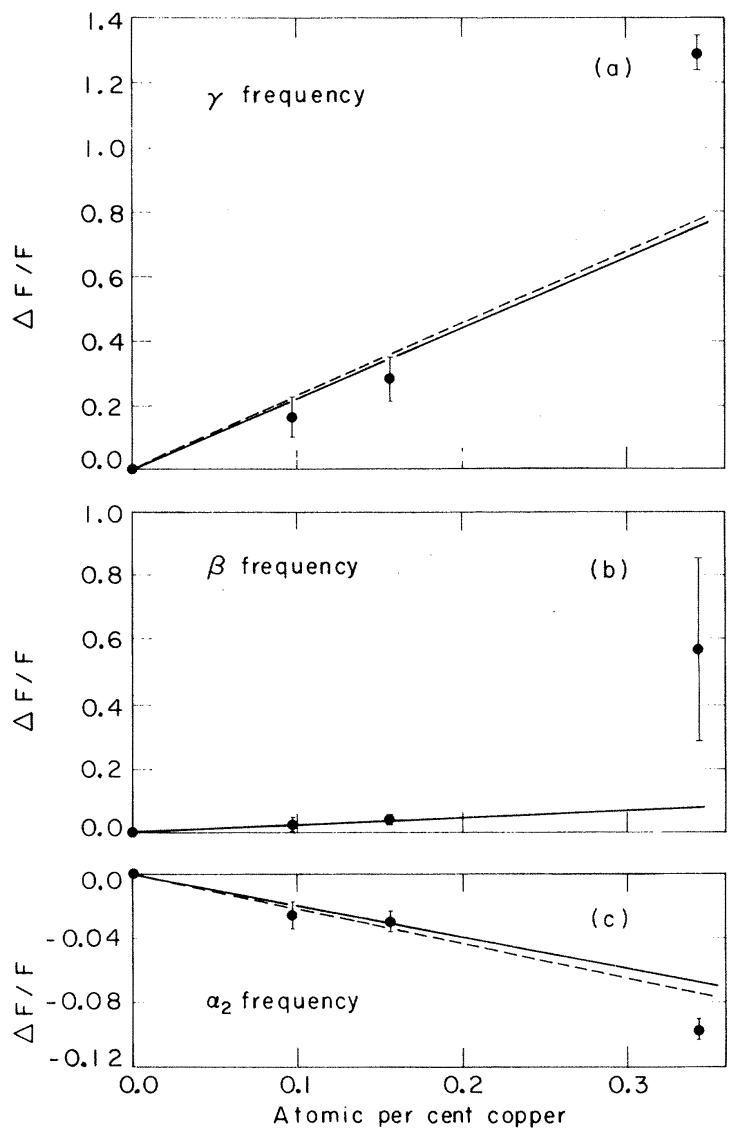

FIG. 2. $\Delta F / F$ versus $\mathrm{Cu}$ concentration for $\alpha_{2}, \beta$, and $\gamma$ frequencies. The solid lines are RBM predictions. The dashed lines include corrections for lattice parameter changes from strain data.

concentration limit.

From Fig. 2 it can be seen that the experimental frequencies in the highest-concentration alloy deviate significantly from the values obtained using the RBM. Table II summarizes this deviation in the 0.34 at. $\% \mathrm{Cu}$ alloy, where $D$, the fractional deviation from the RBM value, is defined as

$$
D \equiv\left(\Delta F-\Delta F_{\mathrm{RBM}}\right) / \Delta F_{\mathrm{RBM}} \text {. }
$$

Since the RBM as formulated by Heine ${ }^{9}$ does not take into account changes in lattice parameters on alloying, it is natural to ask whether such corrections are able to explain the deviations from the RBM seen in Table II. There are two methods for estimating corrections due to lattice-parameter changes. The first of these is based upon the NFE approximation while the second relies on experimental measurements of Fermi-surface area changes with strain.

Harrison ${ }^{38}$ has shown using the NFE approxima- tion for the FS of hexagonal-close-packed metals that the basal plane cross section of the needle piece of this Fermi surface is very dependent on the lattice parameters as well as on the electron concentration. Higgins and Marcus, ${ }^{14}$ using a slightly different geometric approximation, have extended Harrison's work in zinc and have shown how in zinc-copper alloys this effect reversed the direction of the change in the needle cross section with alloying from that predicted by the RBM.

In $\mathrm{Be}$ the only piece of the Fermi surface which is close to the NFE model is the cigar. ${ }^{39}$ Since this corresponds to the nearly-free-electron needle piece of the Fermi surface, the treatment of Higgins and Marcus was used to calculate the change in area of the $\alpha_{2}$ orbit. It was found that the value of the $\alpha_{2}$ cross-sectional area decreased by $1.3 \%$. When this correction to the experimental value is made, $D$ drops from 0.43 to 0.20 , as seen in Table II.

In the second approach, the change in the dHvA frequency due to the change in lattice parameters is calculated using the equation

$$
\Delta F=\frac{\partial F}{\partial a} \Delta a+\frac{\partial F}{\partial c} \Delta c
$$

The value of $\partial F / \partial a$ and $\partial F / \partial c$ for the $\alpha$ and $\gamma$ frequencies can be calculated from the values of $\partial \ln F / \partial \epsilon_{i}$ given in Ref。40, where $\epsilon_{i}$ is the strain in the $i$ th direction。 No strain derivatives were given for the $\beta$ frequency. The dashed lines in Fig. 2 represent these corrections to the RBM. The values used for the lattice parameters in both of these approaches were obtained by first interpolating between the lattice parameters of pure $\mathrm{Be}^{19}$ and those of a 0.65 at. \% alloy. ${ }^{41}$ Then the values were extrapolated to low temperature assuming the same temperature dependence found in pure $\mathrm{Be}^{4}{ }^{42}$

This second approach reduces $D$ for the $\alpha_{2}$ frequency from 0.43 to 0.34 compared to 0.20 for the first method. For the $\gamma$ frequency, $D$ is reduced from 0.70 to 0.68 . In either approach it does not appear that the changes in the lattice parameters

TABLE II. Deviation of 0.34 at. $\% \mathrm{Cu}$ alloy from RBM. $D_{\text {expt }}$ is experimental value. $D_{N}$ is the value corrected for changes in lattice parameters using NFE approximation to Fermi surface (after Ref. 14). $D_{S}$ is the value corrected for changes in lattice parameters using strain derivatives of Ref. 40.

\begin{tabular}{lccccc}
\hline Orbit & $(\Delta F / F)_{\mathrm{RBM}}$ & $(\Delta F / F)_{\text {expt }}$ & $D_{\text {expt }}$ & $D_{N}$ & $D_{S}$ \\
\hline$\alpha_{2}$ & -0.068 & -0.097 & 0.43 & 0.20 & 0.34 \\
$\beta$ & 0.082 & 0.57 & $6.0 \pm 5.0$ & $\cdots$ & $\cdots$ \\
$\gamma$ & 0.76 & 1.29 & 0.70 & $\cdots$ & 0.68 \\
\hline \hline
\end{tabular}


are able to account entirely for the deviation of the highest-concentration alloy from the RBM. It is clear, however, that more data in the higher-concentration region as well as measurements rather than estimates of the lattice parameters of the alloys at low temperatures are needed to come to definite conclusions about the possible failure of the RBM in the more concentrated alloys. The major conclusion, then, of this part of the work is that in the low-concentration limit, where the effects of lattice changes are negligible, there are no deviations from the RBM within the accuracy of our data.

\section{B. Cyclotron Masses}

The cyclotron mass ratio $m_{c}$ for each of the extremal cross sections shown in Fig. 1 was obtained using the measured temperature dependence of the dHvA amplitude $A(T)$ at a given field $B$. The amplitude was iteratively least-squares fit to the expression

$$
\ln \left(\frac{A(T) \sinh \left(C m_{c} T / B\right)}{\left[T \exp \left(C m_{c} T / B\right)\right]}\right)=-C m_{c} T / B+K,
$$

where $C$ is a known constant, ${ }^{43} K$ is a constant independent of $T$, and for the first iteration

$$
\frac{\sinh \left(C m_{c} T / B\right)}{\exp \left(C m_{c} T / B\right)}=\frac{1}{2} \text {. }
$$

Up to 10 temperature points were taken at each field and this was done for as many as 20 field points for each frequency. The cyclotron mass values obtained were averaged over the field range. One of the problems encountered in measuring the cyclotron mass by this method was that second and higher dHvA harmonics could introduce significant errors. This effect was minimized by taking data at as low a field and as high a temperature as was consistent with a good signal-to-noise ratio. Portions of the cyclotron mass plots which deviated significantly from a straight line were rejected.

These results for the four frequencies measured are listed in Table III along with the results of previous work. Within the experimental error of approximately $4 \%$, the cyclotron mass was found to be constant as a function of alloying. It can be seen from Table III that the values of the cyclotron

TABLE III. Cyclotron masses obtained from dHvA data.

\begin{tabular}{lccc}
\hline \hline \multicolumn{4}{c}{ data. } \\
Orbit & $\begin{array}{c}\text { Watts } \\
\text { (Ref. 24) }\end{array}$ & $\begin{array}{c}\text { Tripp et al. } \\
\text { (Ref. 23) }\end{array}$ & Present work \\
\hline$\alpha_{1}$ & 0.17 & $0.168 \pm 0.005$ & $0.165 \pm 0.007$ \\
$\alpha_{2}$ & 0.17 & $0.170 \pm 0.005$ & $0.175 \pm 0.007$ \\
$\beta$ & & $-0.260 \pm 0.009$ & $-0.250 \pm 0.010$ \\
$\gamma$ & & $-0.0212 \pm 0.0006$ & $-0.0220 \pm 0.0008$ \\
\hline \hline
\end{tabular}

mass which have been determined in the present investigation are in very good agreement with previous experiments.

\section{Scattering Temperatures}

The scattering or Dingle temperature $T_{D}$ which appears in the amplitude of the dHvA effect is a measure of the total scattering rate for electrons on a given cyclotron orbit. $T_{D}$ is related to a relaxation time $\tau$ by the equation ${ }^{7}$

$$
T_{D}=\hbar / 2 \pi k \tau \text { 。 }
$$

The interest in $T_{D}$ measurements via the dHvA effect stems from the fact that by such measurements one can obtain information on the anisotropy of the relaxation time over the Fermi surface. Such information is not easily obtainable from bulk-transport measurements.

$T_{D}$ was determined by measuring the dHvA amplitude $A$ as a function of a field at fixed temperature. A least-squares fit of this data was made to the equation

$$
\ln \frac{A B^{1 / 2} \sinh \left(C m_{c} T / B\right)}{J_{2}(\alpha)}=-\frac{C m_{c} T_{D}}{B}+K,
$$

where $K$ is a constant independent of $B, C$ is a known constant, $J_{2}$ is the second-order Bessel function, and $\alpha=2 \pi F B_{m} / B^{2}$, where $B_{m}$ is the modulation amplitude. ${ }^{33,43}$ This was done at four to ten temperatures and the resulting scattering temperatures were averaged. Table IV gives the scattering temperature and relaxation time thus found for both types of nominally pure Be samples and for several frequencies.

The data of Table IV indicate clearly the presence of more than one scattering mechanism. For example, the resistance ratio $\rho\left(300{ }^{\circ} \mathrm{K}\right) / \rho\left(4.2{ }^{\circ} \mathrm{K}\right)$ for $B 0$ was more than twice that for $B 1$; yet the relaxation times for the two samples, determined from the dHvA effect, differed only by about $20 \%$. This result can be rationalized in terms of a predominantly isotropic-scattering mechanism plus a predominantly small-angle scattering mechanis m. The scattering temperature is sensitive to all types of scattering but, as is well known, the residual resistivity is relatively insensitive to small-angle scattering. ${ }^{44}$ Now impurity atoms tend to cause rather isotropic scattering ${ }^{44}$ while dislocations tend to cause small-angle scattering. ${ }^{10,45}$ Thus, the above-mentioned scattering temperature data for the $B 0$ and $B 1$ samples is consistent with roughly equal concentrations of small-angle scatterers while $B 0$ appears to have a considerably lower concentration of isotropic scatterers, presumably impurities.

Figure 3 shows the concentration dependence of $T_{D}$ for three frequencies. In view of the above 
TABLE IV. Scattering temperature for pure Be.

\begin{tabular}{cccc}
\hline \hline Sample & Orbit & $T_{D}\left({ }^{\circ} \mathrm{K}\right)$ & $\tau\left(10^{-12} \mathrm{sec}\right)$ \\
\hline$B 0$ & $\alpha_{2}$ & 2.24 & 0.543 \\
$B 1$ & $\alpha_{2}$ & 2.78 & 0.437 \\
$B 1$ & $\beta$ & 2.50 & 0.486 \\
$B 1$ & $\gamma$ & 2.35 & 0.517 \\
\hline \hline
\end{tabular}

discussion, it seems reasonable to attribute a large part of the scatter in the data to differences in the concentration of small-angle scatterers. For example, although alloys $B 3$ and $B 4$ were made from the same pure $\mathrm{Be}$ ingot, alloy $B 2$ was made from a different ingot at a different time and, thus, may have contained very different secondary impurities and a different amount of vacancies, dislocations, and other lattice defects. The error bars shown in Fig. 3 indicate only the random error 。

It is clear that, since we are interested here in how the conduction electrons interact with charged impurity atoms, it is necessary to separate from the total scattering only that part due to this mechanism. Thus, for measuring the anisotropy in $\tau$ or for comparison with theory, the quantity

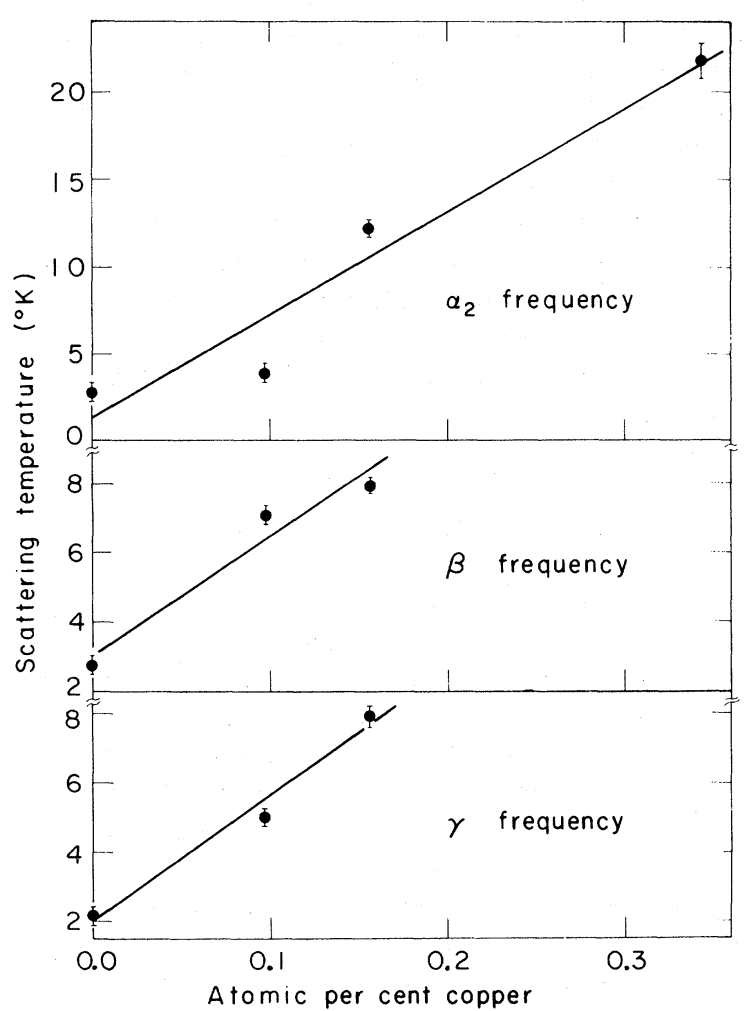

FIG. 3. $T_{D}$ versus $\mathrm{Cu}$ concentration for $\alpha_{2}, \beta$, and $\gamma$ frequencies. $\xi \equiv d \tau^{-1} / d c$ is the important quantity rather than $\tau$ itself. This follows since the reciprocal relaxation time due to impurity scattering is proportional to the concentration in the dilute limit. The values of $d T_{D} / d c$ listed in Table $\mathrm{V}$ were obtained from Fig. 3 and, since $d T_{D} / d c \propto \xi$, it is obvious that there is a considerable anisotropy in the scattering rate for the various orbits studied. This suggests that the copper impurity atoms scatter electrons on the $\alpha_{1}$ and $\alpha_{2}$ cigar orbits considerably more strongly than electrons on the $\beta$ and $\gamma$ orbits. The velocity vectors for the $\alpha_{1}$ and $\alpha_{2}$ are always parallel to the basal plane, while the velocity vectors of the $\beta$ and $\gamma$ orbits are in a plane perpendicular to the basal plane. Thus, it is interesting that, for the orbits studied, electrons in beryllium traveling parallel to the basal plane seem to be scattered more strongly than those traveling in planes parallel to the hexagonal axis.

It is not clear whether the anisotropy in scattering is a reflection of an anisotropic-impurity potential or of the different natures of the Bloch states associated with the various orbits. As yet there have been no theoretical calculations of the scattering rate of electrons by impurities for various Bloch states in a real metal with an anisotropic Fermi surface. Stern ${ }^{6}$ has given a qualitative discussion of this problem, and Seeger and co-workers ${ }^{46}$ have set up the problem in terms of Wannier functions and Green's functions. However Brailsford ${ }^{7}$ has derived a specific formula relating the dHvA relaxation time $\tau$ to the conductivity relaxation time $\tau_{\rho}$ given by

$$
\tau_{\rho}=m / N e^{2} \rho,
$$

where $N$ is the free-electron concentration and $\rho$ the resistivity. He assumed scattering of free electrons by a screened Coulomb potential of the form

$$
V_{p}(r)=-(Z e / r) e^{-q r},
$$

where the screening radius $q^{-1}$ is given by

$$
q^{2}=4 \pi e^{2} n\left(E_{F}\right) .
$$

By considering only charged impurity scattering, he showed that

$$
\tau / \tau_{\rho}=\Phi\left(1+q^{2} / 2 k_{F}^{2}\right),
$$

TABLE V. Summary of scattering temperature data.

\begin{tabular}{lccc}
\hline \hline Orbit & $\begin{array}{c}d T_{D} / d c \\
\left({ }^{\circ} \mathrm{K} / \mathrm{at} . \% \mathrm{Cu}\right)\end{array}$ & $\xi_{\rho} / \xi$ & $\Phi\left(1+q^{2} / 2 k_{F}\right)$ \\
\hline$\alpha_{1}$ & 58.9 & $1.5 \pm 0.2$ & 0.55 \\
$\alpha_{2}$ & 52.8 & $1.6 \pm 0.2$ & 0.55 \\
$\beta$ & 34.0 & $2.8 \pm 0.6$ & 0.55 \\
$\gamma$ & 35.9 & $2.6 \pm 0.6$ & 0.55 \\
\hline \hline
\end{tabular}


where $k_{F}$ is the Fermi radius and where $\Phi$ is given by

$$
\Phi(\lambda)=(\lambda-1)\left[\frac{1}{2}(\lambda+1) \ln \left(\frac{\lambda+1}{\lambda-1}\right)-1\right] .
$$

As mentioned above, in our case which includes some extraneous scattering, we may obtain a measure of the impurity scattering due to impurity atoms only by defining $\xi$ as above and $\xi_{\rho} \equiv d \tau_{\rho}^{-1} / d c$. This gives

$$
\xi_{\rho} / \xi=\Phi\left(1+q^{2} / 2 k_{F}^{2}\right) .
$$

Figure 4 gives the quantity $\xi_{\rho}$ for a sample whose current axis $\vec{J}$ was parallel to [0001]. A similar plot with $\vec{J} ॥[11 \overline{2} 0]$ was shifted upward slightly but had the same slope within experimental error. Table V gives the left-hand side of Eq. (11) as determined from linear least-squares fits to the data in Figs. 3 and 4. The room-temperature electrical resistivities were obtained from the paper by Gruneisen and Erfling. ${ }^{47}$ Also tabulated is the calculated value of $\Phi\left(1+q^{2} / 2 k_{f}^{2}\right)$ which was obtained assuming free-electron values, appropriate to beryllium, for $q$ and $k_{f}$. It is seen that the agreement between experiment and this simple theory based on free electrons is rather poor. Obviously, there is a need for more theoretical work on this problem, both as regards the order of magnitude and the anisotropy of the relaxation time over the Fermi surface.

\section{Long-Period Oscillation}

In the amplitude analysis of the $\alpha$ frequencies performed in order to determine the scattering temperatures, a low-frequency oscillation was

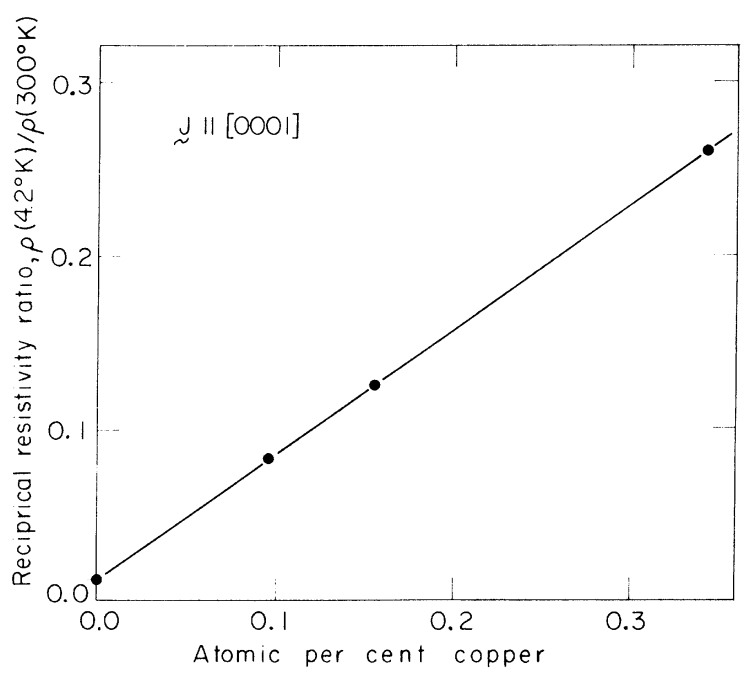

FIG. 4. Reciprocal resistivity ratio versus $\mathrm{Cu}$ concentration for $J \|[0001]$. observed about the least-squares line which determined $T_{D}$. Although the oscillation was observed in both the $\alpha_{1}$ and $\alpha_{2} T_{D}$ plots, in the discussion which follows only the $\alpha_{2}$ frequency results will be considered since the $\alpha_{1}$ frequency data is complicated by magnetic-breakdown effects. Figure 5(a) shows the oscillation in the amplitude of the $\alpha_{2}$ frequency about the linear least-square fit. In Figure $5(\mathrm{~b})$ the $\log$ of the ratio of the experimental amplitude to the linear least-squares amplitude is plotted. Since at most $1 \frac{1}{2}$ oscillations were observed, it was not possible to rigorously determine the periodicity in $1 / B$. More cycles of this low-frequency oscillation were not observed because its period was so long and the $\alpha_{1}$ and $\alpha_{2}$ amplitudes on which it rode were not observable below about $25 \mathrm{kG}$. However, the apparent frequency for $\vec{B} \|[0001]$ in pure beryllium was $\sim 5.4 \times 10^{4} \mathrm{G}$.

This long-period oscillation was seen, in order of decreasing amplitude, in the $B 0, B 1, B 2$, and $B 3$ samples. It was not seen in the highest copper-concentration alloy $B 4$. In one of the pure $\mathrm{Be}$ samples, $B 1$, this oscillation was observed as a function of angle about the [0001] direction in a $(11 \overline{2} 0)$ plane. It was only observable out to $24^{\circ}$ from [0001] since beyond this angle other frequencies came into the beat pattern and the beat period between $\alpha_{1}$ and $\alpha_{2}$ became so long that analysis was not possible with the existing experimental equipment. The apparent frequency dependence of this oscillation as a function of copper concentration and angle is shown in Fig. 6.

Because of the rather regular decrease in frequency with copper concentration, we were tempted initially to consider this frequency as being due to overlap of the cigars with the fourth zone. This overlap was suggested by Watts but has never been observed experimentally nor predicted theoretically. However, in an earlier investigation of the magnetic-interaction effect in beryllium, Condon $^{25}$ had observed some amplitude anomalies in a crystal with $\sim 0.5^{\circ}$ microstructure and no anomalies in a crystal with $\sim 0.1^{\circ}$ microstructure. This led us to consider such an explanation of our "low frequency" and an x-ray investigation in our laboratories showed, in fact, that our crystal did contain $1^{\circ}$ microstructure. ${ }^{48}$ A calculation based on the angular dependence of the $\alpha$ oscillations for $\vec{B}$ near [0001] gave an expected frequency of $\sim 4 \times 10^{4} \mathrm{G}$ in rough agreement with the observed low frequency. Both Figs. 6(a) and 6(b) are consistent with this hypothesis and it, therefore, seems reasonable to attribute the long-period oscillation to microstructure although it is surprising that the frequency varies, within experimental error, in such a regular way in different crystals of 

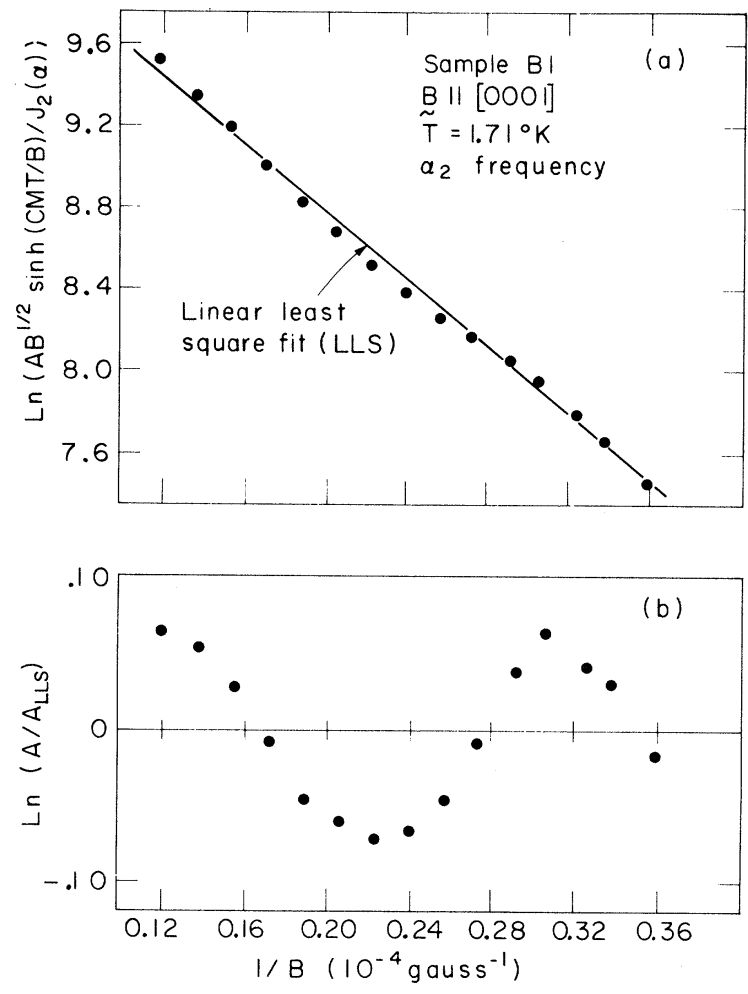

FIG. 5. Low-frequency oscillation as observed in $\alpha_{2}$ amplitude analysis.

various copper concentrations.

\section{SUMMARY AND CONCLUSIONS}

The dHvA effect was used to study the Fermi surface in pure beryllium and beryllium-copper alloys having concentrations of up to 0.34 at. $\%$ $\mathrm{Cu}$. Four extremal areas were chosen which characterize the Fermi surface, the two $\alpha$ cross sections which are associated with the electron cigars, the $\beta$ cross section which is associated with the corner tetrahedron of the hole coronet, and the $\gamma$ cross section associated with the necks of the hole coronets. For copper concentrations up to 0.16 at. $\% \mathrm{Cu}$, the decrease in the electron sheet and increase in the hole sheet with alloying was found to follow the rigid-band model of alloying. However, in the 0.34 at. $\% \mathrm{Cu}$ alloy the Fermi surface was found to consistently change by an amount significantly greater than that predicted by the rigid-band model. When corrections due to lattice-parameter changes were introduced, part of the deviation from the RBM was accounted for。 It was concluded that further measurements of areas and lattice parameters at higher concentrations (between $\sim 0.2$ and $\sim 1.0$ at. $\% \mathrm{Cu}$ are needed to test the theory of Fermi-surface changes due to electronic effects of alloying. In an alloy like Be $(\mathrm{Cu})$, in which the valence difference between solute and solvent is appreciable compared to the valence of the solvent, first-order perturbation theory is not expected to be entirely correct. ${ }^{3}$ Thus, it would be very surprising if the RBM (after lattice-parameter corrections) were valid in $\mathrm{Be}(\mathrm{Cu})$ to a high degree of accuracy in view of the free-electron screening and simple perturbation theory on which it is based.

The cyclotron masses for these four extremal areas have also been determined and have been found to agree very well with previous work. ${ }^{23,24}$ Within the experimental error, no change in the cyclotron mass with alloying was observed.

The scattering temperatures for these extremal areas as a function of alloying were also measured. The derivatives of the reciprocals of the relaxation time derived from these temperatures with respect to concentration were compared with values obtained from the relaxation times derived from the residual-resistance ratio. The ratio

$$
\frac{d \tau_{\rho}^{-1}}{d c} / \frac{d \tau^{-1}}{d c}
$$

thus obtained, was found to be three to five times
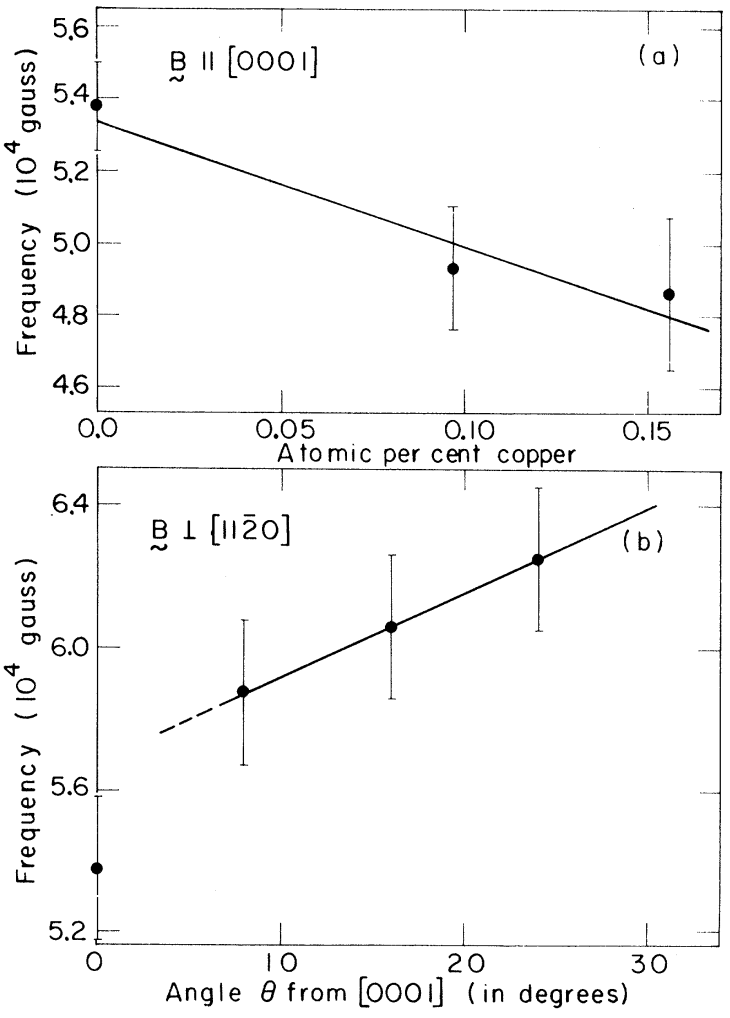

FIG. 6. Low frequency versus (a) Cu concentration, and (b) field direction. 
larger than the theoretical value predicted on the basis of scattering by a Thomas-Fermi potential. ${ }^{7}$

The change in scattering temperature upon alloying was found to be anisotropic. The analysis indicates that electrons on the $\alpha_{1}$ and $\alpha_{2}$ cigar orbits are scattered considerably more strongly than those on the $\beta$ and $\gamma$ coronet orbits. It would be interesting to see whether this behavior could be understood in terms of band-structure effects. A theoretical calculation would involve a numerical solution of the Fermi-Thomas equation for the case of $\mathrm{Be}(\mathrm{Cu})$ and the use of a scattering analysis with numerically calculated wave functions。 ${ }^{49}$

In the analysis of the field dependence of the amplitude of the $\alpha_{2}$ oscillations, a very long-period oscillation $\left(F \simeq 5.4 \times 10^{4} G\right)$ was observed. This frequency was attributed to a beating between $\alpha$ oscillations originating from various parts of the sample, which contained approximately $1^{\circ}$ of microstructure.
* Research supported by the Advanced Research Projects Agency and by the Air Force Materials Laboratory, Wright-Patterson Air Force Base, Ohio; it was performed in part at the Francis Bitter National Magnet Laboratory, Massachusetts Institute of Technology, Cambridge, Mass., which is supported by the United States Air Force Office of Scientific Research.

$\nmid$ Present address: Xerox Corporation, Rochester, N. Y.

${ }^{1}$ H. Jones, Proc. Roy. Soc. (London) A144, 225 (1934).

${ }^{2}$ J. Friedel, Advan. Phys. 3, 446 (1954).

${ }^{3}$ E. A. Stern, Phys. Rev. 144,545 (1966).

${ }^{4}$ E. A. Stern, Phys. Rev. 157,544 (1967).

${ }^{5}$ E. A. Stern, Phys. Rev. 168,730 (1968).

${ }^{6}$ E. A. Stern, Phys. Rev. $\underline{188}, 1163$ (1969).

${ }^{7}$ A. D. Brailsford, Phys. Rev. 149, 456 (1966).

${ }^{8}$ R. B. Dingle, Proc. Roy. Soc. (London) A211, 517 (1952).

${ }^{9}$ V. Heine, Proc. Phys. Soc. (London) A69, 505 (1956).

${ }^{10}$ P. E. King-Smith, Phil. Mag. 12, 1123 (1965).

${ }^{11}$ L. F. Chollet and I. M. Templeton, Phys. Rev. 170 , 656 (1968)

${ }^{12}$ N. B. Brandt and L. G. Lyubutina, Zh。 Eksperim. i Teor. Fiz. 47, 1711 (1964) [ Soviet Phys. JETP 25 450 (1967)]

${ }^{13}$ R. N. Bhargava, Phys. Rev. 156, 785 (1967).

${ }^{14}$ R. J. Higgins ar.: J. A. Marcus, Phys. Rev. 141 , $553(1966)$.

${ }^{15} \mathrm{~J}$. P. G. Shepherd and W. L. Gordon, Phys. Rev. 169, 541 (1968).

${ }^{16}$ R. J. Higgins, H. D. Kaehn, and J. H. Condon, Phys. Rev. 181, 1053 (1969).

${ }^{17}$ W. L. Gordon, J. P. G. Shepherd, and J. Fiske, Bull. Am. Phys. Soc. 12, 331 (1967); P. M. Evcrett, J. M. Fiske, and W. L. Gordon, ibid. 15, 295 (1970); Phys. Rev. $B$ (to be published).

${ }^{18} \mathrm{~J}$. R. Boyd and W. L. Gordon, Bull. Am. Phys. Soc. 12, 331 (1967).

${ }^{19}$ International Tables for X-Ray Crystallography, edited by K. Lonsdale (Kynoch Press, Birmingham, England, 1962), Vol. III.

${ }^{20}$ C. Herring and A. G. Hill, Phys. Rev. 58, 132 (1940)

${ }^{21}$ T. L. Loucks and F. H. Cutler, Phys. Rev. 133, A819 (1964).

${ }^{22}$ J. H. Terrell, Phys. Rev. 149, 526 (1966).

${ }^{23}$ J. H. Tripp, P. M. Everett, W. L. Gordon, and R. W. Stark, Phys. Rev. 180, 669 (1969).

${ }^{24}$ B. R. Watts, Proc. Roy. Soc. (London) A282, 521 (1964).
${ }^{25}$ J. H. Condon, Phys. Rev. 145, 526 (1966).

${ }^{26}$ R. D. Plummer and W. L. Gordon, Phys. Rev. Letters 13, 432 (1964).

${ }^{27}$ N. E. Alekseevskii, V. S. Egorov, and A. V. Dubrovin, Zh. Eksperim. i Teor. Fiz. Pis'ma Redaktsiya 6, 793 (1967) [Soviet Phys. JETP Letters $\underline{6}, 249$ (1967)].

${ }^{28} \mathrm{~N}$. E. Alekseevskii and V. S. Egorov, Zh. Eksperim. i Teor. Fiz. 45, 388 (1963)[Soviet Phys. JETP 18, 268 (1964)]

${ }^{29}$ N. E. Alekseevskii and V. S. Egorov, Zh. Eksperim. i Teor. Fiz. 46, 1205(1964) [Soviet Phys. JETP 19, 815 (1964)].

${ }^{30}$ I. S. Goldstein and P. J. Tobin, Rev. Sci. Instr. 40, 172 (1969).

${ }^{31} \mathrm{~J}$. Ahn and D. J. Sellmyer, Phys. Rev. B 1,1285 (1970).

${ }^{32}$ A. C. Thorsen and T. G. Berlincourt, Rev. Sci. Instr. 34, 435 (1963).

${ }^{33}$ See, for example, A. Goldstein, S. J. Williamson, and S. Foner, Rev. Sci. Instr. 36, 1356 (1965); L. R. Windmiller and J. B. Ketterson, ibid. 39, 1672 (1968); R. W. Stark and L. R. Windmiller, Cryogenics 8, 272 (1968).

${ }^{34}$ I. S. Goldstein, Ph. D. thesis, Massachusetts Institute of Tecnnology, 1969 (unpublished).

${ }^{35} \mathrm{~N}$. W. Asheroft and J. W. Wilkins, Phys. Letters 14, 285 (1965).

${ }^{36} \mathrm{Cee}$, for example, the review article by C. P. Enz, in Theory of Condensed Matter (International Atomic Energy Agency, Vienna, 1968), p. 729.

${ }^{37}$ G. Ahlers, Phys. Rev. 145, 419 (1966).

${ }^{38}$ W. A. Harrison, Phys. Rev. 118, 1190 (1960).

${ }^{39}$ See Ref. 24, Fig. 9.

${ }^{40}$ B. S. Chandrasekhar, E. Fawcett, D. M. Sparlin, and G. K. White, in Proceedings of Tenth International Conference on Low-Temperature Physics, Moscow, 1966 (Proizvodstrenno-Izdatel'skii Kombinat, VINITI, Moscow, 1967), p. 328.

${ }^{41}$ F. Witt, V. V. Damiano, and G. London, Rev. Sci. Instr. 38, 1069 (1967).

${ }^{42}$ R. W. Meyerhoff and J. P. Smith, J. Appl. Phys. 33, 219 (1962).

${ }^{43}$ I. M. Lifshitz and A. M. Kosevich, Zh. Eksperim. i Teor. Fiz. 29, 730 (1956) LSoviet Phys. JETP 2,636 (1 956)].

${ }^{44} \mathrm{~A}$. B. Pippard, The Dynamics of Conduction Electrons (Gordon and Breach, New York, 1965), p. 44.

${ }^{45}$ P. G. Klemens and J. L. Jackson, Physica 31, 1421 (1965).

${ }^{46}$ For a review and references, see A. Seeger, Comments Solid State Phys. 11, 82 (1969). 


\footnotetext{
${ }^{47}$ E. Gruneisen and H. D. Erfling, Ann. Physik 38, $399(1940)$

${ }^{48} \mathrm{R}$. Curratt (private communication).

${ }^{49}$ E. A. Stern (private communication).
}

\title{
Diffusivity and Isotope-Effect Measurements in Equiatomic Fe-Co ${ }^{\dagger *}$
}

\author{
S. G. Fishman, $\$$ D. Gupta, $\S^{\S}$ and D. S. Lieberman \\ Department of Metallurgy and Mining Engineering, and Materials Research Laboratory, \\ University of Illinois, Urbana-Champaign, Illinois 61801
}

(Received 29 December 1969)

\begin{abstract}
Several radioactive isotopes of $\mathrm{Fe}$ and $\mathrm{Co}$ were simultaneously diffused into an equiatomic FeCo alloy which exhibits fcc, bcc, and CsCl-type ordered phases with decreasing temperature. Penetration profiles were obtained by standard lathe sectioning and a grinding-sectioning technique described in this paper. From $\ln D$-versus $-1 / T$ plots, activation energies of 68.5 and $55 \mathrm{kcal}$ and frequency factors of 1.26 and $0.25 \mathrm{~cm}^{2} / \mathrm{sec}$ were obtained for diffusion of $\mathrm{Fe}^{59}$ in the fcc and bcc phases, respectively. From similar plots, activation energies of 69.4 and $60.0 \mathrm{kcal}$ and frequency factors of 1.33 and $2.00 \mathrm{~cm}^{2} / \mathrm{sec}$ were obtained for diffusion of $\mathrm{Co}^{57}$ in the fcc and bcc phases of the FeCo alloy. The average isotope-effect parameters were 0.66 in the fcc phase and 0.52 in the bcc phase. Manning's theory for concentrated alloys was used to calculate correlation factors for the FeCo alloy. From these calculated values and experimentally measured isotope-effect parameters, $\Delta K$ was determined to be slightly larger in the fcc and bcc phases of the FeCo alloy than has been reported in pure $\mathrm{Fe}$. The observed decrease in the isotope-effect parameter with increasing degree of order during the bcc-to-CsCl-type ordering transformation is explained in terms of an increase in atom-jump correlation with the onset of long-range order.
\end{abstract}

\section{INTRODUCTION}

The predominant mechanism of diffusion in cubic metals and dilute alloys has been identified as atomvacancy interchange. Since there is a certain probability that a diffusing atom will jump back into the vacancy and cancel out the effect of the jump on mass diffusion, its motion is described by a Bardeen-Herring-type correlation. ${ }^{1}$ The correlation factor is the fraction of atom jumps which is effective in contributing to random diffusion and is intimately related to the diffusion mechanism. In pure metals it is a simple geometric factor which has been calculated theoretically and deduced from measurements. " The so-called "isotope-effect" measurement is useful for determining correlation factors and distinguishing among mechanisms of diffusion. While measurements of the isotope effect in self-diffusion have been made in a number of pure metals and dilute alloys, ${ }^{3,4}$ little work has been reported in concentrated binary alloys. Since most materials in everyday use are composed of concentrated alloys, it is important to establish the mechanisms of self-diffusion in such materials and to understand the degree and nature of correlations in these mechanisms.

The present investigation was undertaken to measure self-diffusion, correlation, and isotope effects in concentrated alloys. The previous studies in this laboratory were on self-diffusion in highly ordered near-equiatomic $\beta$-AuCd ${ }^{5,6}$ and $\beta$-AuZn ${ }^{6,7}$ alloys as a function of composition and temperature; pressure effects were also investigated in the latter. ${ }^{6}$ However, no correlation studies were made in these systems. Generally, tracer diffusion and correlation effects in nondilute alloys cannot be treated as rigorously as in pure metals and dilute alloys. The approach in such cases has been limited to two idealized models: the completely random alloy and the completely ordered alloy. In random alloys, it is assumed that the vacancy exchanges with tracer atoms occur with their characteristic jump frequencies independent of the configuration of the surrounding atoms, and the correlation of the atom jumps is of the Bardeen-Herring type. Manning ${ }^{8,9}$ has developed a simplified model for diffusion kinetics in random concentrated alloys which yields equations demonstrating the primary effects to be expected. In the case of ordered alloys, the problem can be reduced to a reasonably simple form, since the alloy may be considered to consist of two interpenetrating sublattices where each sublattice is occupied by atoms of one kind. The primary constraint on vacancy motion in the ordered lattice is the requirement of maintaining long-range order.

Three diffusion mechanisms have been proposed for atomic motion in CsCl-type ordered alloys which leave the degree of long-range order un- 